\title{
Differential effects of photobiomodulation interval schedules on brain cytochrome c-oxidase and proto-oncogene expression
}

\author{
Jorge L. Arias, ${ }^{\text {a,b }}$ Marta Mendez $\oplus^{\text {a,b }}$ Juan Ángel Martínez, ${ }^{\text {,,c }}$ \\ and Natalia Arias $\odot^{b, d, *}$ \\ ${ }^{a}$ University of Oviedo, Neuroscience Laboratory, Department of Psychology, Oviedo, Spain \\ bINEUROPA, Instituto de Neurociencias del Principado de Asturias, Oviedo, Spain \\ ${ }^{\mathrm{c}}$ University of Oviedo, Escuela Politécnica de Gijón, Departamento Ingeniería Eléctrica, \\ Electrónica, Computadores y Sistemas, Gijón, Spain \\ dinstitute of Psychiatry, Psychology and Neuroscience, Maurice Wohl Clinical Neuroscience \\ Institute, King's College London, Department of Basic and Clinical Neuroscience, \\ London, United Kingdom
}

\begin{abstract}
Significance: Transcranial photobiomodulation (PBM) is a noninvasive neuromodulation technique capable of producing changes in the mitochondrial cytochrome c-oxidase (CCO) activity of neurons. Although the application of PBM in clinical practice and as a neurophysiological tool is increasing, less is known about how different treatment time intervals may result in different outcomes.

Aim: We evaluated the effects of different PBM treatment intervals on brain metabolic activity through the $\mathrm{CCO}$ and proto-oncogene expression (c-Fos).

Approach: We studied PBM effects on brain CCO and c-Fos expression in three groups of animals: Control (CN, $n=8)$, long interval PBM treatment (LI, $n=5)$, and short interval PBM treatment (SI, $n=5$ ).

Results: Increased CCO activity in the LI group, compared to the SI and CN groups, was found in the prefrontal cortices, dorsal and ventral striatum, and hippocampus. Regarding c-Fos expression, we found a significant increase in the SI group compared to LI and CN, whereas LI showed increased c-Fos expression compared to $\mathrm{CN}$ in the cingulate and infralimbic cortices.

Conclusions: We show the effectiveness of different PBM interval schedules in increasing brain metabolic activity or proto-oncogene expression.

(C) The Authors. Published by SPIE under a Creative Commons Attribution 4.0 Unported License. Distribution or reproduction of this work in whole or in part requires full attribution of the original publication, including its DOI. [DOI: 10.1117/1.NPh.7.4.045011]
\end{abstract}

Keywords: photobiomodulation; cytochrome c-oxidase; c-Fos; striatum; prefrontal cortex.

Paper 20048R received Jun. 5, 2020; accepted for publication Oct. 28, 2020; published online Dec. 8, 2020.

\section{Introduction}

Transcranial photobiomodulation (PBM) is a noninvasive neuromodulation technique that has the ability to increase cellular metabolism and blood flow. It can be utilized for neuroprotection due to its role in reversing apoptotic signaling processes, and it has been found to promote synaptogenesis, among other actions. ${ }^{1}$ Although the precise cellular and molecular mechanisms underlying PBM are not yet fully understood, light in the 600- to 1200-nm wavelength range has significant PBMT (photobiomodulation therapy) capability. ${ }^{2}$

*Address all correspondence to Natalia Arias, natalia.arias@kcl.ac.uk 


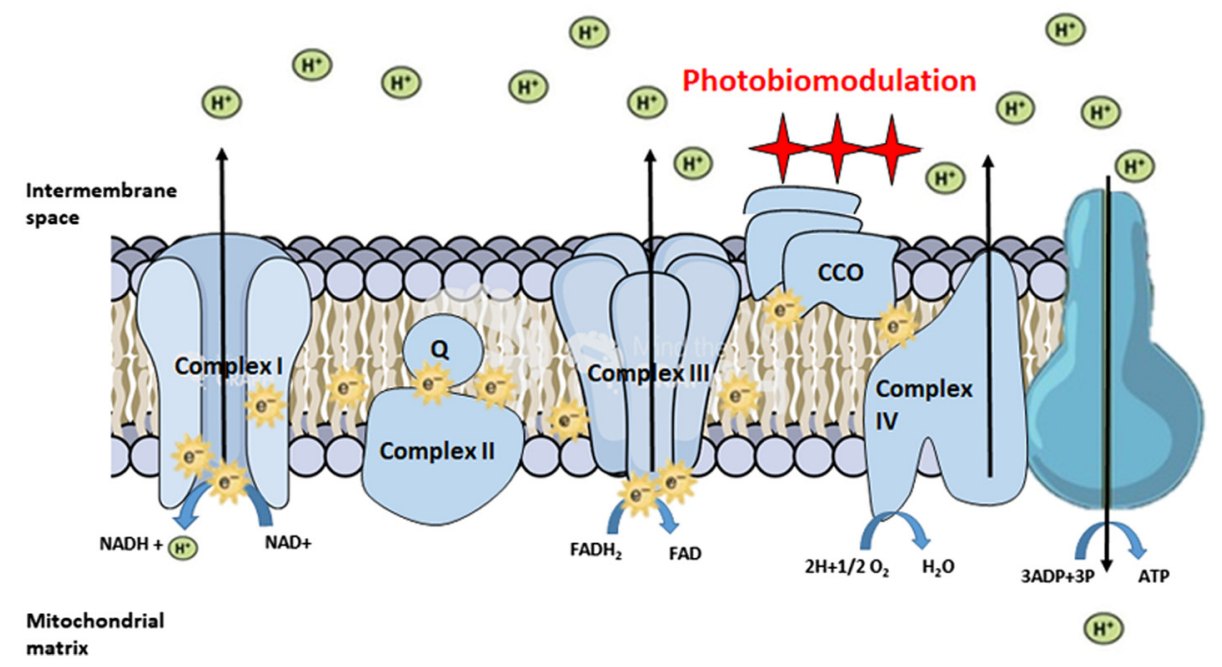

Fig. 1 Mechanisms of action in PBMT strategies. PBM directly stimulates CCO (complex IV), facilitating its catalytic activity and inducing an increase in holoenzyme subunit assembly.

Transcranial PBMT is based on photon energy absorption and upregulation of cytochrome c-oxidase $(\mathrm{CCO}),{ }^{3,4}$ which has resulted in neuroprotective effects in traumatic brain injury, ${ }^{5}$ ischemic stroke, ${ }^{6}$ Alzheimer's disease,${ }^{7}$ Parkinson's disease, ${ }^{8}$ and psychological disorders such as depression and anxiety, ${ }^{9,10}$ as well as in age-related cognitive decline. ${ }^{11,12}$ Within PBMT, low-level light therapy is a new therapeutic technology that interacts with CCO inside the mitochondria, restoring electron transport chain activity, ${ }^{13,14}$ and therefore, improving energy metabolism (Fig. 1).

However, the effects on the brain of different time intervals between individual treatments have not been explored, although there is some evidence suggesting that this is an important parameter in other PBMT applications. ${ }^{15}$ Animal studies have demonstrated that a single PBMT administration is able to reduce cognitive and motor deficits in a traumatic brain injured (TBI) animal model. ${ }^{16,17}$ In this line, Cassano et al. $(2015),{ }^{18}$ using higher power $(5 \mathrm{~W})$ laser diodes for short intervals, were able to remit depressive symptoms in patients. Conversely, in humans with chronic or mild TBI, the only treatments that showed beneficial effects were long interval treatments performed three times a week for 6 weeks. ${ }^{19}$ Similarly, treatment for major depressive disorder is effective when administered twice a week for 8 weeks. ${ }^{20}$ Thus, the effects of different intervals (e.g., long versus short) of PBMT treatments on the brain have not been explored.

Another current debate in the field is whether pulsing or continuous light could have an effect of penetration depth and, hence, a differential effect on target activation, such as CCO. Studies by Henderson and Morries ${ }^{2}$ have shown that pulsed light yielded greater penetration through sheep skin, intact sheep heads, and living human tissue, but these results were not statistically significant and resulted in overall lower irradiance compared to continuous light. Conversely, on fixed human cadaver heads, there was no difference between pulse- and continuous-wave laser light. ${ }^{21}$ However, in animal TBI studies, pulse-wave laser light was superior to continuous-wave, ${ }^{22}$ and it was found to avoid unpleasant heat damage due to continuous irradiation periods. ${ }^{23,24}$

Our aim was to evaluate the effect of different intervals (long versus short) of PBMT on neural activity through c-Fos immunohistochemistry and CCO histochemistry. It was recently shown that PBM affects the production of reactive oxidative species and increases intracellular $\mathrm{Ca}^{2+},{ }^{25}$ which can initiate the Ras/extracellular signal-regulated kinase (ERK) cascade, ${ }^{26}$ producing long-lasting effects on cells and suggesting the potential expression of some proto-oncogenes such as c-Fos. However, the effect of PBM on proto-oncogene expression has not been explored.

The intracellular transcription of c-Fos will need an adenosine triphosphate (ATP) source, and so we wanted to explore energy metabolism through the study of CCO activity. For this purpose, we measured CCO activity through optical densitometry. It is well known that almost all the energy obtained in neurons is derived from oxidative phosphorylation in the mitochondria, and CCO is one of the key energy generating enzymes in this process. The PBM action 
mechanism is based on photon energy absorption and upregulation of cytochrome oxidase, ${ }^{3,4}$ which has resulted in behavioral and metabolic neuroprotection in animal models of retinal neurotoxicity, ${ }^{27,28}$ traumatic brain injury, ${ }^{29}$ and autoimmune encephalomyelitis. ${ }^{30}$ We hypothesized that PBMT would increase neuronal respiration and boost brain energy metabolic capacity, ${ }^{31}$ which could be reflected in an increase in brain regional CCO activity.

\section{Materials and Methods}

\subsection{Subjects}

We used 18 male Wistar rats (290 to $330 \mathrm{~g}$ ). They were maintained under standard laboratory conditions $\left(20^{\circ} \mathrm{C}\right.$ to $22^{\circ} \mathrm{C}, 65 \%$ to $70 \%$ relative humidity, and a 12 -h light/dark cycle). All procedures were carried out according to the European Parliament and the Council of the European Union 2010/63/UE and approved by the Oviedo University committee for animal studies. The animals were randomly distributed into three groups, one control and two differential interventions: control group (CN, $n=8)$, long interval PBMT group (LI, $n=5)$, and short interval PBMT group (SI, $n=5$ ).

\subsection{Photobiomodulation Therapy}

Animals in the long interval PBMT group (LI) received 1 session of low-light level treatment a day for 7 days (between 9:00 and 10:00 a.m.). Animals in the short interval PBMT group (SI) received four PBMT sessions for a total of 3 min within $30 \mathrm{~h}^{32}$ The administration of the light in the SI group took place at 10:00 a.m., 17:30 p.m., 01:00 a.m., and 8:30 a.m. PBMT was delivered by a $670 \pm 10$-nm wavelength LED array (Quantum Devices Warp 10, Barneveld), the device $\left(5.1 \mathrm{~cm}\right.$ diameter, $1073 \mathrm{~mW}, 50 \mathrm{~mW} / \mathrm{cm}^{2}$ ) delivered for $3 \mathrm{~min}$ a total of $9 \mathrm{~J} / \mathrm{cm}^{2}$. The device was placed on the midline of the dorsal surface of the animal's shaved head in the region between the eyes and ears. To avoid PBMT to other brain regions, an opaque material covered the rest of the device; moreover, in order to assure consistency, the same researcher delivered the treatment in all groups and conditions. The equipment used to measure the power of the light source was a PM160 Optical Power Meter by THORLABS (New Jersey). Animals in the control group (CN) were subjected to the same protocol, except that the LED device was turned off.

\subsection{Brain Processing}

All the animals were decapitated 90 min after the last PBMT exposure. Then, the brains were removed intact, frozen rapidly in isopentane (Sigma-Aldrich, Germany), and stored at $-40^{\circ} \mathrm{C}$. We use 90-min postprobe because the cellular c-fos proto-oncogene is an immediate early expression gene whose induction is one of the first cellular responses after the application of a variety of stimuli. This induction is rapid and transient and can encode its protein with a peak in expression between 1 and $2 \mathrm{~h}^{33,34}$

Coronal sections $(30 \mu \mathrm{m})$ of the brain were cut at $-20^{\circ} \mathrm{C}$ in a cryostat (Leica CM1900, Germany). Distance in $\mathrm{mm}$ of brain regions counted from bregma was: $+3.20 \mathrm{~mm}$ for anterior cortices, including the infralimbic (IF), prelimbic (PL), and cingulate (CG) cortex; $+1.2 \mathrm{~mm}$ for the dorsal striatum (STR), including the anterodorsal, anterolateral, and anteromedial; $+1.2 \mathrm{~mm}$ for the accumbens core (ACC) and accumbens shell (ACS); and $-3.3 \mathrm{~mm}$ for the cornu ammonis (CA)1, CA3, and dentate gyrus (DG) subareas of the dorsal hippocampus. The selected brains regions were anatomically defined according to the atlas by Paxinos and Watson. ${ }^{35}$

\subsubsection{Cytochrome c-oxidase histochemistry}

The protocol used was the same one previously described. ${ }^{36}$ Briefly, sets of tissue homogenate standards from Wistar rat brains were included with each bath of slides. Sections and standards were incubated in 0.1 phosphate buffer (PB) with $10 \%(\mathrm{w} / \mathrm{v})$ sucrose and $0.5(\mathrm{v} / \mathrm{v})$ glutaraldehyde. Then, baths of $0.1 \mathrm{M}$ PB with sucrose were given. Subsequently, 0.05M Tris buffer was 
applied. Then, sections and standards were incubated in a solution with $0.0075 \%$ cytochrome-c $(\mathrm{w} / \mathrm{v}), 0.002 \%$ catalase (w/v), $5 \%$ sucrose $(\mathrm{w} / \mathrm{v}), 0.25 \%$ dimethylsulfoxide $(\mathrm{v} / \mathrm{v})$, and $0.05 \%$ diaminobenzidine tetrahydrochloride in $0.1 \mathrm{M} \mathrm{PB}$. The reaction was stopped by fixing the tissue in buffered $4 \%(\mathrm{v} / \mathrm{v})$ formalin. Finally, the slides were dehydrated and cleared with xylene. CCO histochemical staining intensity was quantified by densitometric analysis using a computer-assisted image analysis workstation (MCID, Interfocus Imaging Ltd., Linton, England). A total of 12 measurements were taken per brain region. These measures were averaged to obtain one mean per region for each animal, and they were expressed as arbitrary units of optical density (OD).

\subsection{2 c-Fos activity}

Five animals from each group were processed immunocytochemically for c-Fos. The sections were mounted on gelatinized slides. Then, the sections were post-fixed in buffered $4 \%$ paraformaldehyde (0.1M, pH 7.4) for $30 \mathrm{~min}$ and rinsed in phosphate-buffered saline (PBS) $(0.01 \mathrm{M}$, $\mathrm{pH}$ 7.4). They were subsequently incubated for $15 \mathrm{~min}$ with $3 \%$ hydrogen peroxidase in PBS to remove endogenous peroxidase activity and then washed twice in PBS. After blocking with PBS solution containing 10\% Triton X-100 (PBS-T) (Sigma, USA) and 3\% bovine serum albumin for $30 \mathrm{~min}$, sections were incubated with a rabbit polyclonal anti-c-Fos solution (1:10.000) (Santa Cruz Biotech, sc-52, USA) diluted in PBS-T for $24 \mathrm{~h}$ at $4^{\circ} \mathrm{C}$ in a humid chamber. Slides were then washed three times with PBS and incubated in a goat anti-rabbit biotinylated IgG secondary antibody (Pierce, USA; diluted 1:200 in incubating solution) for $2 \mathrm{~h}$ at room temperature. They were washed three times in PBS and reacted with avidin-biotin peroxidase complex (Vectastain ABC Ultrasensitive Elite Kit, Pierce) for $1 \mathrm{~h}$. After two washes in PBS, the reaction was visualized, treating the sections for about $3 \mathrm{~min}$ in a commercial nickel-cobalt intensified diaminobenzidine kit (Pierce). The reaction was finalized by washing the sections twice in PBS. Slides were then dehydrated through a series of graded alcohols, cleared with xylene, and cover-slipped with Entellan (Merck, USA) for microscopic observation. All the immunocytochemistry procedures included sections that served as controls where the primary antibody was not added. Slides containing sections of a specific brain region were stained at the same time. Slides were coded so that the investigator who performed the entire analysis would have no knowledge of the group to which the individual subjects belonged.

The total number of c-Fos positive nuclei was quantified in three alternate sections $30 \mu \mathrm{m}$ apart. Quantification was done by systematically sampling each of the regions selected, using a microscope (Olympus BH-2, Japan) attached to an analog camera (Sony XC-77, Japan) and a TV monitor $(300 \times$ total magnification). c-Fos-positive nuclei were defined based on homogeneous gray-black stained elements with a well-defined border. Finally, the mean count for three sections was calculated for each subject and region (number of positive nuclei/150 $\mu \mathrm{m}^{2}$ ).

\subsection{Statistical Analysis}

All data were analyzed by the Sigma-Stat 3.2 program (Systat, Richmond) and expressed as the mean $\pm \mathrm{SEM}$. The results were considered statistically significant if $p<0.05$. A one-way repeated measures of analysis of variance (ANOVA) was used for the statistical comparison of the CCO activity and c-Fos expression values between the groups. Post-hoc multiple comparison analyses were carried out when possible, using pairwise Tukey tests. Moreover, a nonparametric Kruskall-Wallis test $(\mathrm{H})$ for independent samples was performed when normality or equal group variances failed.

\section{Results}

\subsection{CCO Activity}

When we explored the CCO brain activity, we found higher activity in the LI group compared to the other groups in PL $(\mathrm{F} 2,17=9.699, p=0.002)$ between SI $(p=0.002)$ and $\mathrm{CN}(p=0.028)$, 
Arias et al.: Differential effects of photobiomodulation interval schedules...
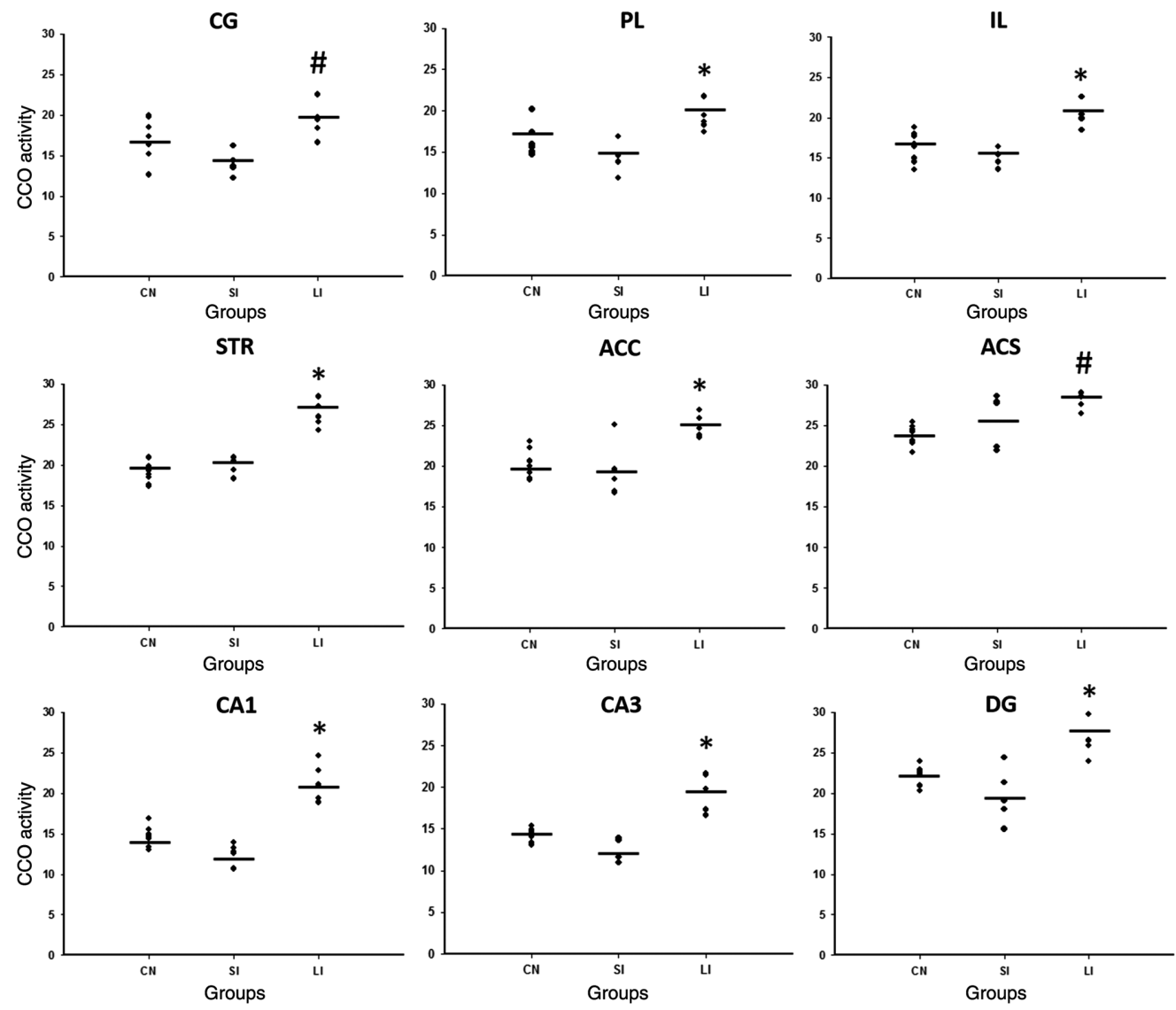

Fig. 2 CCO histochemistry in the sampled regions where significant differences were found in cingulate (CG), prelimbic (PL) and infralimbic (IL) cortices, dorsal striatum (STR), ACC, ACS, $\mathrm{CA} 1, \mathrm{CA} 3$, and dentate gyrus (DG) subregions of the hippocampus. ${ }^{*} p<0.05$ versus SI and $\mathrm{CN}$ groups. Lines represent mean.

IL (F2,17 $=15.980, p<0.001)$ compared to SI $(p<0.001)$ and CN $(p=0.002)$, STR (F2,17 = $49.566, p<0.001)$ compared to SI $(p<0.001)$ and CN $(p<0.001)$, ACC $(\mathrm{F} 2,17=9.335$, $p=0.002)$ compared to SI $(p=0.003)$ and $\mathrm{CN}(p=0.007)$, CA1 (F2,17 $=41.314, p<$ $0.001)$ compared to SI $(p<0.001)$ and CN $(p<0.001)$, CA3 (F2,17 $=27.543, p<0.001)$ compared to SI $(p<0.001)$ and $\mathrm{CN}(p<0.001)$, and DG (F2,17 = 12.407, $p<0.001)$ compared to SI $(p<0.001)$ and CN $(p=0.005)$. Moreover, increased CCO activity in CG $(\mathrm{F} 2,17=7.750$, $p=0.005)$ was found between LI compared to the SI group ( $p=0.004)$ (Fig. 2).

\section{2 c-Fos Activity}

We found a significant increase in proto-oncogene expression, c-Fos, in the SI group compared to LI and CN. One-way ANOVA showed significant differences in PL (F2,14 $=46.233$, $p<0.001)$ compared to LI $(p<0.001)$ and $\mathrm{CN}(p<0.001)$, IL (F2,14 = 16.944, $p<0.001)$ compared to LI $(p=0.026)$ and $\mathrm{CN}(p<0.001), \mathrm{CG}(\mathrm{F} 2,14=17.912, p<0.001)$ compared to LI $(p=0.046)$ and $\mathrm{CN}(p<0.001)$, STR $(\mathrm{H} 2=10.220, p=0.006)$ compared to $\mathrm{CN}$ $(p<0.05)$, ACC (F2,14 = 17.350, $p<0.001)$ compared to LI $(p<0.001)$ and $\mathrm{CN}(p=0.006)$, ACS (F2,14 $=9.717, p=0.003)$ compared to LI $(p=0.011)$ and CN $(p=0.004)$, and CA3 $(\mathrm{F} 2,14=12.185, p=0.001)$ compared to LI $(p=0.002)$ and $\mathrm{CN}(p=0.004)$. Moreover, differences were found between LI and CN in IL $(p=0.042)$ and CG $(p=0.017)$. Furthermore, increased c-Fos expression was found in the SI group in comparison with LI $(p=0.011)$ in CA1 (F2,14 $=6.401, p=0.013)$. Finally, no differences between the groups were found in DG (F2,14 = 0.861, $p=0.447)$ (Fig. 3). 

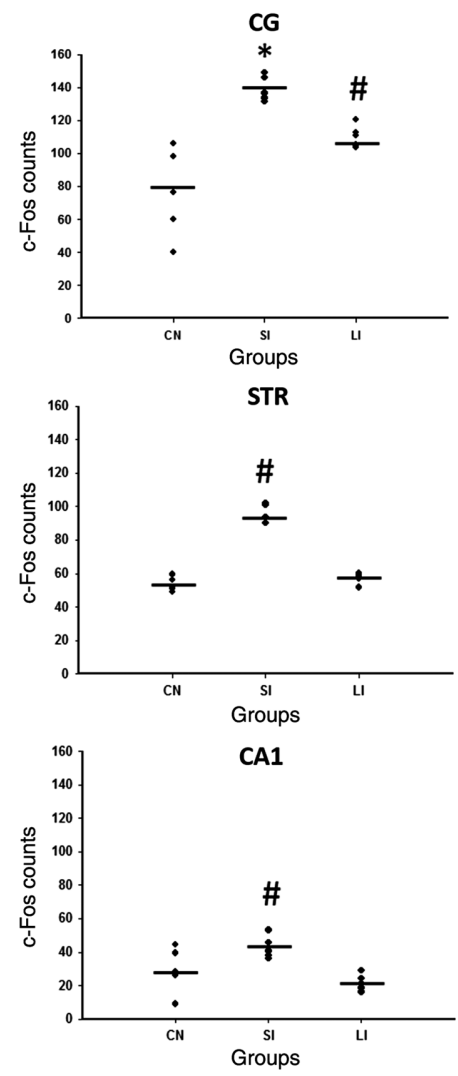
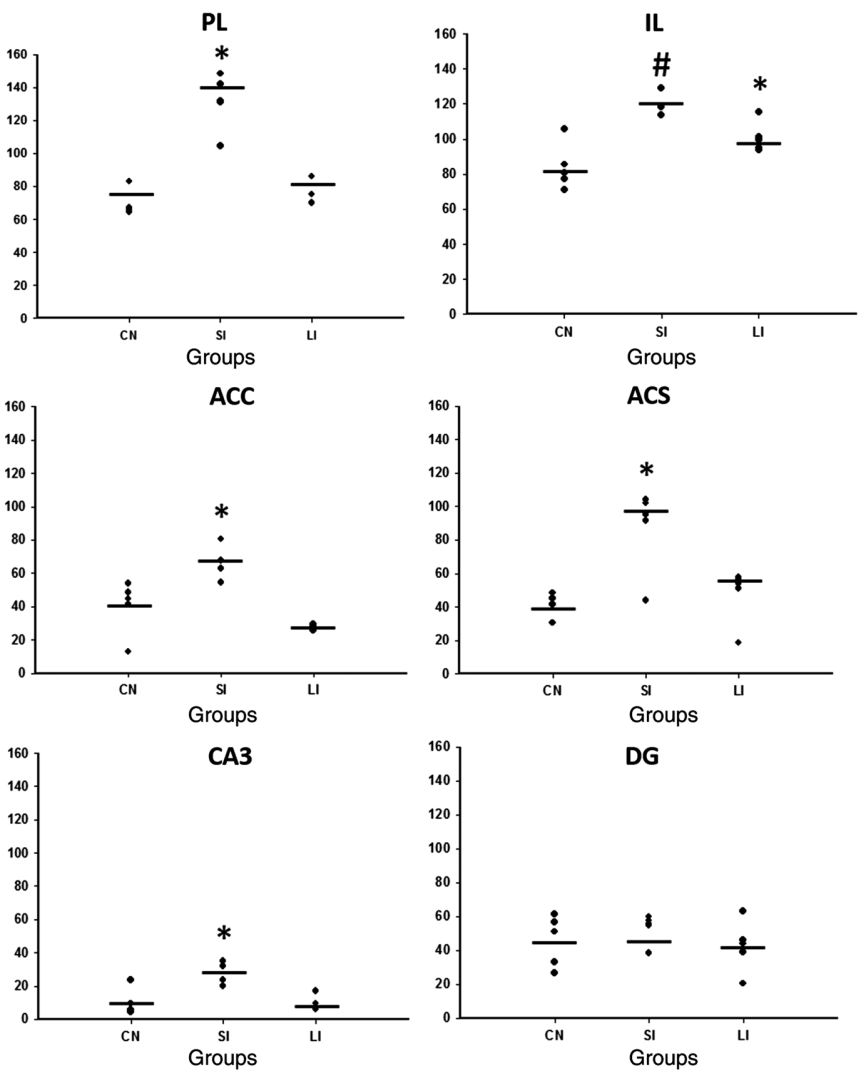

Fig. 3 c-Fos immunoreactivity expression in the sampled regions where significant differences were found in cingulate (CG), prelimbic (PL) and infralimbic (IL) cortices, dorsal striatum (STR), ACC, ACS, CA1, CA3 subregions of the hippocampus. ${ }^{*} p<0.05$ versus $\mathrm{SI}$ and $\mathrm{CN}$ groups. $\# p<0.05$ versus. $C N$ group. No differences were found in the dentate gyrus (DG). Lines represent mean.

\section{Discussion}

Transcranial PBM involves exposing cells or tissues to low level light photons in the wavelength range from red to near-infrared light (600 to $1200 \mathrm{~nm}$ ). ${ }^{37} \mathrm{PBM}$ is an emerging therapeutic technology that interacts with $\mathrm{CCO}$ inside the mitochondria, restoring the electron transport chain activity, ${ }^{13,14}$ and therefore, improving energy metabolism. Transcranial PBM is based on photon energy absorption and upregulation of $\mathrm{CCO},{ }^{3,4}$ which improves neuronal metabolic capacity in the rat brain, ${ }^{31}$ increased ATP content, ${ }^{38}$ and altered mitochondrial dynamics. ${ }^{39}$ Moreover, transcranial PBM has produced neuroprotective effects in several disorders, such as traumatic brain injury, ${ }^{5}$ ischemic stroke,${ }^{6}$ Alzheimer's disease, ${ }^{7}$ Parkinson's disease, ${ }^{8}$ and anxiety and depression. ${ }^{9,10}$ This treatment also shows promising results in improving cognitive decline related to aging. ${ }^{11,12}$ However, despite its promising role as a therapeutic agent, less is known about the impact of different intervals of PBMT treatment on the brain function.

Our results demonstrated that PBMT was able to boost brain metabolism not only by increasing mitochondrial $\mathrm{CCO}$ activity but also through the activation of proto-oncogene expression. Moreover, we found a differential brain effect depending on the intervals between successive stimulations (short versus long intervals).

Indeed, the LI group showed increased CCO activity in all the hippocampal subregions, infralimbic and prelimbic cortices, striatum, and accumbens nucleus, compared to the SI and $\mathrm{CN}$ groups. These results could be explained by the biological photoacceptor nature of CCO ${ }^{28,40}$ Specifically, absorption of the photons delivered in PBM seems to promote an increase in the availability of electrons for the reduction of molecular oxygen in the catalytic center of $\mathrm{CCO}$, thus increasing the mitochondrial membrane potential, ATP levels, and reactive oxygen species, which leads to increased mitochondrial function. ${ }^{41}$ 
Furthermore, it has been shown that PBM is able to photodissociate NO from $\mathrm{CCO},{ }^{42}$ which could reverse the mitochondrial inhibition of cellular respiration that exists as a result of excessive NO binding. ${ }^{43}$ Thus, NO would no longer compete with oxygen in binding to the catalytic metal centers of $\mathrm{CCO}$, allowing for an influx of oxygen. Therefore, enzymatic activity and respiration could return to baseline, increasing CCO activity and ATP production, which is in line with our findings.

Another interesting finding is the general activation found in the brain regions studied. The relationship between the hippocampus and other brain regions in supporting memory processes is well known. Regarding this, the cooperation between the hippocampus and the striatum has been reported during episodic encoding. ${ }^{44,45}$ The episodic memory network comprises the cingulate cortex ${ }^{46,47}$ and medial prefrontal cortex, ${ }^{48}$ as well as the aforementioned regions, highlighting that they belong to an established network. Indeed, it has been suggested that when transcranial light irradiation coincides geographically with the stimulation of some brain regions involved in any intrinsic network, a therapeutic benefit will be extended to the entire brain network. ${ }^{49}$ In this line, a systematic effect of low-PBMT has been previously described by Naeser et al. $^{50}$ where benefit to cerebral perfusion was observed when PBMT was applied to an acupuncture point on the foot. These systematic attributes to PBMT have been supported by changes in nitric oxide levels ${ }^{51}$ and excitotoxic modulation secondary to brain injury. ${ }^{29}$ Moreover, although there is a lack of studies that have tested whether neurotransmission is involved in mediating the protective effects of PBM, it has been demonstrated that PBMT increases endothelial nitric oxide synthase activity ${ }^{52}$ leading to a transient increase in NO, which has an important role in neurotransmission and signal transduction. ${ }^{53,54}$ So, our CCO results could be supporting this systematic effect where activation of some of the elements of the network could trigger the activation of deeper brain regions involved, as it has been demonstrated when PBMT was applied to the default mode network. ${ }^{55,56}$

In fact, this dose fractionation based on long intervals was recently found to produce cognitive improvement, evaluated with the minimental state exam, in mild to moderately severe dementia patients and patients with Alzheimer's disease. ${ }^{57}$ In addition, research has also shown that PBMT fractionation protocols that include prophylactic doses given before neurotoxic metabolic lesions are also effective in preventing neurodegeneration. ${ }^{58}$

Less explored has been the effect of PBMT on proto-oncogene expression, such as c-fos. Our results show that c-Fos expression increased after SI intervention in the CA1 and CA3 areas of the hippocampus, prefrontal cortices, striatum, and accumbens nucleus. c-Fos expression reflects evoked cellular activation following stimuli application, ${ }^{59}$ such as, in our case, PBMT administration. We hypothesized that this increased c-Fos expression could be explained by two complementary mechanisms. First, the ability of low-level light to activate mitogen-activated protein kinases (MAPK) phosphorylation initiates the ERK cascade until activating c-Fos expression. ${ }^{60}$ Second, it was recently demonstrated that low-light level therapy could contribute to increased intracellular $\mathrm{Ca}^{2+}$, which is a versatile second messenger ${ }^{61,62}$ involved in transcriptional regulation via protein kinase A (PKA), MAPK, and calmodulin-stimulated protein kinase. ${ }^{63}$ Moreover, an increase in $\mathrm{Ca}^{2+}$ intracellular concentration can initiate the Ras/ERK cascade, ${ }^{27}$ followed by increased c-Fos expression. Finally, PBM has been found to trigger retrograde mitochondrial signaling. ${ }^{64}$ This refers to signals and communications passing from the mitochondria to the nucleus of a cell, rather than vice versa. Thus, our findings might suggest that longer administration intervals could be driving the aforementioned communication between mitochondrial changes and the nucleus. ${ }^{65}$ Moreover, it is important to note that a differential increase in cFos expression could be appreciated among brain regions where prefrontal cortices showed the higher rates compared to subcortical regions such as striatum, hippocampus, and accumbens nucleus. These results are in line with Ref. 22, who demonstrated that near-infrared light penetrated the brain and scattered, thus establishing a gradient of light energy as the photons were absorbed and distributed within the brain tissue. So, these results could be supporting the light fluence gradient within the brain.

Finally, although our study with continuous light at different schedules proved different brain effects, other studies have proved that pulsing PBMT was able to change the spiking in parvalbumin n-positive interneurons and reduced the levels of amyloid $\beta$ peptides in the brain of Alzheimer's mice model. ${ }^{66}$ Moreover, Henderson and Morries ${ }^{51,67}$ studied the relevance of 
changes in power densities to achieve sufficient light penetration to the brain. All these results pointed out that future research should focus on how varying treatment parameters can change brain function, and how treatments can potentially be tuned for differing brain ailments.

In conclusion, our results add experimental evidence about the differential effects of PBMT intervals on brain stimulation. LI schedules have been shown to increase CCO activity in many structures that take part in the limbic memory network, whereas SI intervention enables c-Fos expression. This study may facilitate the development of new strategies to boost cortical and subcortical neurocognitive activity. Further studies should be carried out to explore PBMT systemic administration effects following these intervals on the same molecular mechanisms within the neurons.

\section{Disclosures}

The authors have no relevant financial interests in the manuscript and no other potential conflicts of interest to disclose.

\section{Acknowledgments}

This work was supported by the Ministry of Science, Innovation and Universities (PSI201783893-R to JLA and MM) and the Ministry of Economy and Business (PSI2015-73111EXP to JLA, and PSI2017-90806-REDT to JLA) (Spain). The funders had no role in the study design or data collection. We would like to thank the Alzheimer's Research UK King's College London Network Centre and AINDACE foundation for their technical and human support.

\section{Code, Data, and Materials Availability}

The authors have declared that the manuscript does not have associated code. Moreover, the authors have declared the availability of data used in the research results reported in the manuscript and have provided specific access information or restrictions (i.e., persistent weblinks to repository access addresses). Finally, the authors have declared the availability of materials, explained any restrictions on materials (i.e., materials transfer agreements), or provided supplementary materials for the purpose of reproducibility.

\section{References}

1. M. R. Hamblin and Y. Y. Huang, Photobiomodulation in the Brain, Academic Press, San Diego, California (2019).

2. T. A. Henderson and L. D. Morries, "Near-infrared photonic energy penetration: can infrared phototherapy effectively reach the human brain?" Neuropsychiatr. Dis. Treat. 11, 2191-2208 (2015).

3. X. Wang, et al., "Interplay between up-regulation of cytochrome-c-oxidase and hemoglobin oxygenation induced by near-infrared laser," Sci. Rep. 6, 30540 (2016).

4. X. Wang, et al., "Up-regulation of cerebral cytochrome-c-oxidase and hemodynamics by transcranial infrared laser stimulation: a broadband near infrared spectroscopy study," J. Cereb. Blood Flow Metab. 37(12), 3789-3802 (2017).

5. T. Ando et al., "Comparison of therapeutic effects between pulsed and continuous wave 810-nm wavelength laser irradiation for traumatic brain injury in mice," PLoS One 6(10), e262122011 (2011).

6. K. Yip et al., "The effect of low-energy laser irradiation on apoptotic factors following experimentally induced transient cerebral ischemia," Neuroscience 190, 301-306 (2011).

7. L. De Taboada et al., "Transcranial laser therapy attenuates amyloid- $\beta$ peptide neuropathology in amyloid- $\beta$ protein precursor transgenic mice," J. Alzheimers Dis. 23(3), 521-535 (2011).

8. A. Oueslati et al., "Photobiomodulation suppresses alpha-synuclein-induced toxicity in an AAV-based rat genetic model of Parkinson's disease," PLoS One 10(10), e0140880 (2015). 
9. F. Schiffer et al., "Psychological benefits 2 and 4 weeks after a single treatment with near infrared light to the forehead: a pilot study of 10 patients with major depression and anxiety," Behav. Brain Funct. 5(1), 46 (2009).

10. F. Salehpour and S. H. Rasta, "The potential of transcranial photobiomodulation therapy for treatment of major depressive disorder," Rev. Neurosci. 28(4), 441-453 (2017).

11. F. Salehpour et al., "Transcranial low-level laser therapy improves brain mitochondrial function and cognitive impairment in D-galactose-induced aging mice," Neurobiol. Aging $\mathbf{5 8}$, 140-150 (2017).

12. S. Michalikova et al., "Emotional responses and memory performance of middle-aged CD1 mice in a 3D maze: effects of low infrared light," Neurobiol. Learn Mem. 89(4), 480-488 (2008).

13. M. R. Hamblin et al., "Mechanisms and mitochondrial redox signaling in photobiomodulation," Photochem. Photobiol. 94, 199-212 (2018).

14. M. Hennessy et al., "Photobiomodulation and the brain: a new paradigm," J. Opt. 19, 013003 (2018).

15. P. Brondon, I. Stadler, and R.J. Lanzafame, "A study of the effects of phototherapy dose interval on photobiomodulation of cell cultures," Lasers Surg. Med. 36(5), 409-413 (2005).

16. W. Xuan et al., "Transcranial low-level laser therapy enhances learning, memory, and neuroprogenitor cells after traumatic brain injury in mice," J. Biomed. Opt. 19(10), 108003 (2014).

17. H. I. Lee et al., "Pre-conditioning with transcranial low-level light therapy reduces neuroinflammation and protects blood-brain barrier after focal cerebral ischemia in mice," Restor. Neurol. Neurosci. 34(2), 201-214 (2016).

18. P. Cassano et al., "Near-infrared transcranial radiation for major depressive disorder: proof of concept study," Psychiatry J. 2015, 352979 (2015).

19. M. A. Naeser et al., "Significant improvements in cognitive performance post-transcranial, red/near-infrared light-emitting diode treatments in chronic, mild traumatic brain injury: open-protocol study," J. Neurotrauma 31(11), 1008-1017 (2014).

20. P. Cassano et al., "Transcranial photobiomodulation for the treatment of major depressive disorder. The ELATED-2 pilot trial," Photomed. Laser Surg. 36(12), 634-646 (2018).

21. C. E. Tedford et al., "Quantitative analysis of transcranial and intraparenchymal light penetration in human cadaver brain tissue," Lasers Surg. Med. 47(4), 312-322 (2015).

22. A. Oron et al., "Near infrared transcranial laser therapy applied at various modes to mice following traumatic brain injury significantly reduces long-term neurological deficits," J. Neurotrauma 29(2), 401-407 (2012).

23. P. A. Lapchak et al., "Transcranial infrared laser therapy improves clinical rating scores after embolic strokes in rabbits," Stroke 35(8), 1985-1988 (2004).

24. X. Wang et al., "Impact of heat on metabolic and hemodynamic changes in transcranial infrared laser stimulation measured by broadband near-infrared spectroscopy," Neurophotonics 5(1), 011004 (2018).

25. F. Salehpour et al., "Transcranial near-infrared photobiomodulation attenuates memory impairment and hippocampal oxidative stress in sleep-deprived mice," Brain Res. 1682, 36-43, (2018).

26. L. B. Rosen et al., "Membrane depolarization and calcium influx stimulate MEK and MAP kinase via activation of Rats," Neuron 12(6), 1207-1221 (1994).

27. J. C. Rojas, et al., "Neuroprotective effects of near-infrared light in an in vivo model of mitochondrial optic neuropathy," J. Neurosci. 28(50), 13511-13521 (2008).

28. M. T. Wong-Riley et al., "Photobiomodulation directly benefits primary neurons functionally inactivated by toxins: role of cytochrome c oxidase," J. Biol. Chem. 280(6), 4761-4771 (2005).

29. T. Dong et al., "Low-level light in combination with metabolic modulators for effective therapy of injured brain," J. Cereb. Blood Flow Metab. 35(9), 1435-1444 (2015).

30. K. A. Muili et al., "Amelioration of experimental autoimmune encephalomyelitis in C57BL/ 6 mice by photobiomodulation induced by $670 \mathrm{~nm}$ light," PLoS One 7(1), e30655 (2012).

31. J. C. Rojas, A. K. Bruchey, and F. Gonzalez-Lima, "Low-level light therapy improves cortical metabolic capacity and memory retention," J. Alzheimers Dis. 32(3), 741-752 (2012).

32. V. E. Shaw et al., "Neuroprotection of midbrain dopaminergic cells in MPTP-treated mice after near-infrared light treatment," J. Comp. Neurol. 518(1), 25-40 (2010). 
33. W. Tischmeyer and R. Grimm, "Activation of immediate early genes and memory formation," Cell. Mol. Life Sci. 55(4), 564-574 (1999).

34. J. A. Harris, "Using c-fos as a neural marker of pain," Brain Res. Bull. 45(1), 1-8 (1998).

35. G. Paxinos and C. H. Watson, The Rat Brain in Stereotaxic Coordinates-the New Coronal Set, 5th ed., Elsevier Academic Press, San Diego, California (2005).

36. N. Arias, M. Méndez, and J. L. Arias, "The importance of the context in the hippocampus and brain related areas throughout the performance of a fear conditioning task," Hippocampus 25(11), 1242-1249 (2015).

37. H. Chung et al., "The nuts and bolts of low-level laser (light) therapy," Ann. Biomed. Eng. 40(2), 516-533 (2012).

38. T. Karu, L. Pyatibrat, and G. Kalendo, "Irradiation with He-Ne laser increases ATP level in cells cultivated in vitro," J. Photochem. Photobiol. B. 27(3), 219-223 (1995).

39. D. Pastore, et al., "Increase in <-H+/e- ratio of the cytochrome c oxidase reaction in mitochondria irradiated with helium neon laser," Biochem. Mol. Biol. Int. 34(4), 817-826. (1994).

40. T. I. Karu, "Multiple roles of cytochrome c oxidase in mammalian cells under action of red and IR-A radiation," IUBMB Life 62(8), 607-610 (2010).

41. L. F. de Freitas and M. R. Hamblin, "Proposed mechanisms of photobiomodulation or low-level light therapy," IEEE J. Sel. Top. Quantum Electron. 22(3), 7000417 (2016).

42. N. Lane, "Cell biology: power games," Nature 443(7114), 901-903 (2006).

43. S. Moncada and J.D. Erusalimsky, "Does nitric oxide modulate mitochondrial energy generation and apoptosis?" Nat. Rev. Mol. Cell Biol. 3(3), 214-220 (2002).

44. T. Sadeh et al., "Cooperation between the hippocampus and the striatum during episodic encoding," J. Cognit. Neurosci. 23(7), 1597-1608 (2011).

45. S. Frisch et al., "Dissociating memory networks in early Alzheimer's disease and frontotemporal lobar degeneration-a combined study of hypometabolism and atrophy," PLoS One. 8, e55251 (2013).

46. M. Irish, J.R. Hodges, and O. Piguet, "Episodic future thinking is impaired in the behavioural variant of frontotemporal dementia," Cortex 49, 2377-2388 (2013).

47. M. Irish, J. R. Hodges, and O. Piguet, "Right anterior temporal lobe dysfunction underlies theory of mind impairments in semantic dementia," Brain 137, 1241-1253 (2014).

48. M. Irish et al., "Scene construction impairments in Alzheimer's disease-A unique role for the posterior cingulate cortex," Cortex 73, 10-23 (2016).

49. M. A. Naeser et al., "Transcranial, red/near-infrared light-emitting diode therapy to improve cognition in chronic traumatic brain injury," Photomed. Laser Surg. 34(12), 610-626 (2016).

50. M. A. Naeser et al., "Improved cognitive function after transcranial, light-emitting diode treatments in chronic, traumatic brain injury: two case reports," Photomed. Laser Surg. 29(5), 351-358 (2011).

51. T. A. Henderson and L. Morries, "Near-infrared photonic energy penetration: can infrared phototherapy effectively reach the human brain?" Neuropsychiatr. Dis. Treat. 11, 2191-2208 (2015).

52. H. I. Lee et al., "Pretreatment with light-emitting diode therapy reduces ischemic brain injury in mice through endothelial nitric oxide synthase-dependent mechanisms," Biochem. Biophys. Res. Comm. 486, 945-950 (2017).

53. K. Tanaka, "Is nitric oxide really important for regulation of the cerebral circulation? Yes or No?" Keio J. Med. 45, 14-27 (1996).

54. S. Moncada et al., "Nitric oxide: physiology, pathophysiology and pharmacology," Pharmacol. Rev. 43(2), 109-142 (1991).

55. R. L. Buckner, "The serendipitous discovery of the brain's default network," Neuroimage 62(2), 1137-1145 (2012).

56. C. A. Raji et al., "Functional neuroimaging with default mode network regions distinguishes PTSD from TBI in a military veteran population," Brain Imaging Behav. 9(3), 527-534 (2015).

57. A. E. Saltmarche et al., "Significant improvement in cognition in mild to moderately severe dementia cases treated with transcranial plus intranasal photobiomodulation: case series report," Photomed. Laser Surg. 35(8), 432-441 (2017). 
58. Y. Zhuo et al., "Inhibition of bone resorption by the cathepsin K inhibitor odanacatib is fully reversible," Bone 67, 269-280 (2014).

59. D. G. Herrera and H. A. Robertson, "Activation of c-fos in the brain," Prog. Neurobiol. 50(2-3), 83-107 (1996).

60. H. Sohn et al., "Effects of light-emitting diode irradiation on RANKL-induced osteoclastogenesis," Lasers Surg. Med. 47, 745-755 (2017).

61. R. Lavi et al., "Low energy visible light induces reactive oxygen species generation and stimulates an increase of intracellular calcium concentration in cardiac cells," J. Biol. Chem. 278, 40917-40922 (2003).

62. C. C. Lan et al., "Induction of primitive pigment cell differentiation by visible light (heliumneon laser): a photoacceptor-specific response not replicable by UVB irradiation," J. Mol. Med. (Berl) 90, 321-330 (2012).

63. R. D. Fields, P. R. Lee, and J. E. Cohen, "Temporal integration of intracellular $\mathrm{Ca}^{2+}$ signaling networks in regulating gene expression by action potentials," Cell Calcium 37, 433-442 (2005).

64. T. I. Karu, "Mitochondrial signaling in mammalian cells activated by red and near-IR radiation," Photochem. Photobiol. 84(5), 1091-1099 (2008).

65. S. Passarella and T. Karu, "Absorption of monochromatic and narrow band radiation in the visible and near IR by both mitochondrial and nonmitochondrial photoacceptors results in photobiomodulation," J. Photochem. Photobiol. B. 140, 344-358 (2014).

66. H. F. Iaccarino et al., "Gamma frequency entrainment attenuates amyloid load and modifies microglia," Nature 540(7632), 230-235 (2016).

67. T. A. Henderson and L. Morries, "SPECT perfusion imaging demonstrates improvement of TBI with transcranial near infrared laser phototherapy," Adv. Mind Body Med. 29(4), 27-33 (2015).

Jorge L. Arias is a professor at the University of Oviedo. He received his $\mathrm{BS}$ and $\mathrm{PhD}$ degrees in biology from the University Complutense of Madrid in 1981 and 1984. He is the author of more than 250 journal papers and has written more than 20 book chapters. He created the Institute of Neurosciences of the Principality of Asturias in 2011 and has directed it since then. His current research interests include the effects of noninvasive optical and nonoptical techniques on the brain. His laboratory is focused on the cognitive outcomes of these treatments in different neurodegenerative animal models and neurodevelopment.

Marta Mendez received her PhD in psychology from the University of Oviedo, Spain, in 2009. She is currently an associate professor of neuroscience at the University of Oviedo (Department of Psychology). She has authored and coauthored a large number of publications in peerreviewed journals. Her current research interests include neural systems involved in spatial learning and memory and its modification by stimulation.

Juan Ángel Martínez received his $\mathrm{MSc}$ and $\mathrm{PhD}$ degrees in electrical engineering from the University of Oviedo, in Spain, in 1987 and 1991. He joined the University of Oviedo in 1987, having been involved in several research projects. He is currently a professor at this University. His main research interests deal with the design of microcontroller-based sensor and electronic systems often related to medical applications. He is member of the Institute of Neuroscience of Principality of Asturias (INEUROPA).

Natalia Arias is a postdoctoral researcher at King's College London. She earned her International $\mathrm{PhD}$ in neuroscience at the University of Oviedo. She won several grants to continue exploring mechanisms underlying dementia in collaboration with Consiglio Nazionale delle Ricerche, University of Cambridge, and University College of London. Her research is focused on understanding the mechanisms underlying the transition from presymptomatic to symptomatic stages of Amyotrophic Lateral Sclerosis and exploring different therapies to delay the disease using in vitro and in vivo models. She is the author of more than 40 journal papers and has written more than 10 book chapters. 